\title{
Efecto de la información en el proceso de producción y distribución de una empresa de metalmecánica
}

\section{RESUMEN}

La investigación se basa en un juego de negocios conocido como el juego de la cerveza, aplicada a una empresa metalmecánica. La diferencia principal de esta investigación está en la elección de los participantes (personal de la empresa en funciones similares). La metodología empleada en la obtención de datos y los resultados obtenidos en sus diversos escenarios: sin información, demanda conocida, cooperación y comunicación.

Los resultados experimentales del moldeamiento del sistema de la producción y distribución de somier en una empresa de fabricación de muebles metálicos muestran en forma experimental el valor del acceso a la información y su efecto en la rentabilidad y eficacia de la empresa con la entrega de productos y servicios en el momento y lugar correcto, evitando costos innecesarios y el manejo inadecuado de los recursos.

Palabras claves: información, cadena de suministro, bullwhip, dinámica de sistemas.

EFFECT OF THE INFORMATION IN THE PROCESS OF PRODUCTION AND UNA'S DISTRIBUTION METALMECÁNICA'S COMPANY

\section{ABSTRACT}

The research is focused on a business game known as the beer game which was applied to a metal mechanic company. The main difference of this research, with respect to other studies, is the choice of participants (company staff performing similar roles). The methodology applied different scenarios to obtain the data and the results: unknown information, wellknown demand, cooperation and communication. The experimental results in a production and distribution system of a furniture company shows experimentally the value of access to information and its effect on profitability and business efficiency with the delivery of products and services in the time and the right place, avoiding unnecessary costs and inadequate management of resources.

Keywords: information, supply chain, bullwhip, system dynamics.

\section{INTRODUCCIÓN}

La cadena de suministro es un sistema dinámico, no lineal, con diversos controles establecidos por los tiempos, retrasos y los flujos dinámicos (información, productos, materiales y otros).

La investigación plantea la colaboración y aumento de la visibilidad de la información a través de los participantes para mejorar la satisfacción de los clientes, evitar la incertidumbre de los participantes y mitigar la variabilidad y amplitud de las órdenes de producción.

Los resultados experimentales del moldelamiento del sistema de producción y distribución de somier en una empresa de fabricación de muebles metálicos muestran en forma experimental el valor del acceso a la información y su efecto en la rentabilidad y eficacia de la empresa con la entrega de productos y servicios en el momento y lugar correcto, evitando costos innecesarios y el manejo inadecuado de los recursos.

\section{FUNDAMENTOS TEÓRICOS}

La figura 1 tiene los conceptos usando el modelo de hexágonos. El modelo permite visualizar la interdependencia, las relaciones dinámicas y la conexión de los elementos, agrupándolos en procesos similares con sus priorizaciones conceptuales.

Los participantes de una cadena de suministro deberían trabajar en forma conjunta para reducir los costos y satisfacer las expectativas de los clientes con los productos correctos, en el mercado correcto y en el tiempo correcto.

Beneficios:

- Mejora en la satisfacción del cliente con un servicio inmediato de acceso de la información.

- Nuevas oportunidades de ingreso con el incremento de la competitividad y diferenciación de servicios al mostrar la información a los clientes.

- Reducción de los costos operativos.

1 Doctor en Gestión de Empresas - Univ. Nacional Mayor de San Marcos, Máster en Ing. Industrial e Ing. de Sistemas - Universidad de Lima

E-mail: chong.mario@gmail.com

2 Business Object. "Business Intelligence Web Sevice". www.bussinessobject.com. 
Figura 1. Fundamentos teóricos

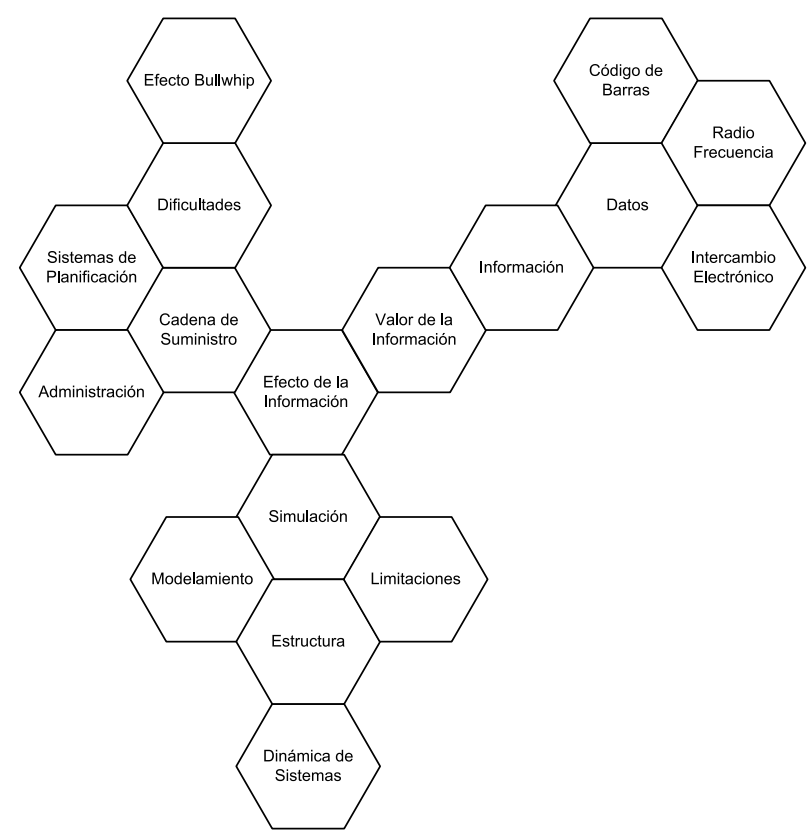

Fuente: elaboración propia.

\section{APLICACIÓN DE LA METODOLOGÍA}

La metodología se apoya en el estudio del manejo de los inventarios, el comportamiento de las órdenes y las diversas políticas que toman los participantes para evitar consecuencias inesperadas e indeseables.

\subsection{MODELAMIENTO}

Las fases para el moldelamiento de un sistema se pueden dividir en:

- Diagramación inicial: descripción del problema con las variables en un espacio de tiempo definido.

- Interpretación formal: diagramación formal con los niveles (rectángulos), flujos (válvulas) y variables auxiliares (círculos).

- Cuantificación de los factores: generación del modelo de simulación y probar la validez del modelo.

Figura 2. Diagramación inicial del modelo

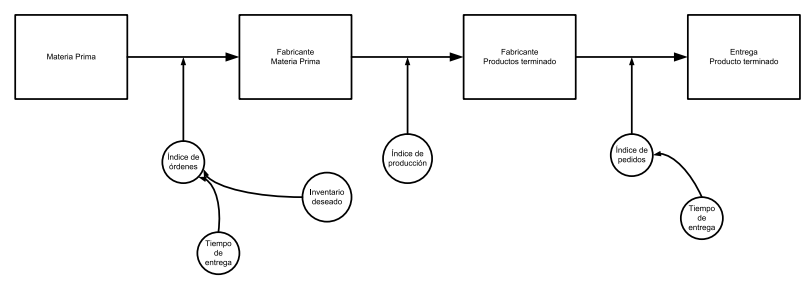

Fuente: elaboración propia.

\subsection{METODOLOGÍA}

La investigación propone usar los conceptos de la dinámica de sistemas para el estudio y el manejo de complejos sistemas de retroalimentación que se pueden encontrar en los negocios y otros sistemas sociales $^{3}$; con sus etapas:

1. Identificación del problema.

2. Desarrollo de la hipótesis, análisis de las posibles causas del problema.

3. Construir un modelo de simulación del sistema:

- Definir los niveles.

- Ilustración de los flujos de retroalimentación.

- Generación del modelo.

4. Prueba del modelo.

5. Desarrollo de posibles alternativas.

6. Implementación de la solución.

Figura 3. Metodología utilizada

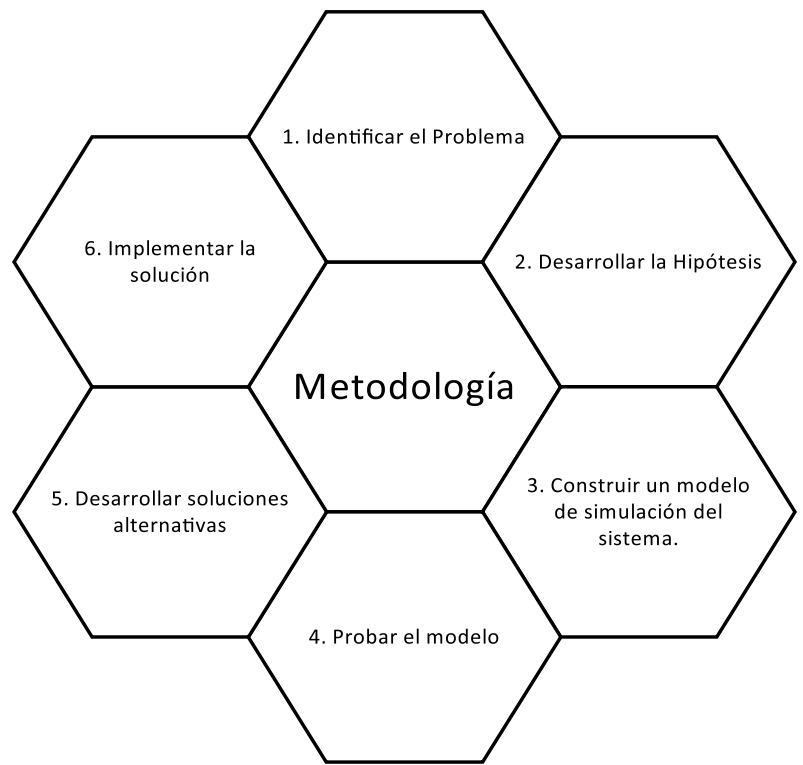

Fuente: elaboración propia

\subsubsection{DESARROLLO DE LA METODOLOGÍA}

El desarrollo de esta sección se va a centrar en la construcción de un modelo experimental con el juego de producción y distribución de la cerveza o beergame.

El juego fue desarrollado en el MIT en 1960 para entender las ventajas de un sistema integrado en una cadena de suministro considerando una cadena de suministro con cuatro participantes: fabricante, distribuidor, mayorista y minorista.

\footnotetext{
3 System Dynamics Society. www.systemdynamics.org
} 
En cada periodo, los participantes tratan de cumplir con la demanda de su cliente, entregando los productos que tiene en el inventario y generando órdenes para futuras entregas. El objetivo final es minimizar el costo de todos los participantes de la cadena de suministro.

\subsection{CONSTRUCCIÓN DE UN MODELO DE SIMULACIÓN DEL SISTEMA}

El modelo inicial tiene como base el juego clásico, sobre el cual se van a desarrollar diversos escenarios con el objetivo de demostrar experimentalmente que existe una variabilidad y amplitud de las órdenes desde el minorista al fabricante.

La diferencia entre los modelos experimentales previos está en los participantes que son personas relacionadas con una empresa metal mecánica, dedicada a la fabricación de muebles metálicos, relacionando la parte experimental con la parte práctica en los experimentos.

\subsubsection{ESCENARIOS}

El modelo experimental va a considerar un modelo de producción-distribución con cuatro participantes (fabricante, distribuidor, mayorista y minorista). El ambiente del modelo maneja en su estructura: los niveles, los flujos de retroalimentación, los flujos, los tiempos de retraso y las relaciones ente sus componentes.

El objetivo de estos escenarios es identificar las políticas para reducir la amplitud y variabilidad de las órdenes desde el cliente hasta el fabricante.

\subsubsection{CONFIGURACIÓN DEL EXPERIMENTO}

Los cuatro participantes en cada periodo van a recibir órdenes de sus clientes, recibir los productos, entregar los productos y generar órdenes a sus proveedores.

Figura 4. Flujo de los principales escenarios

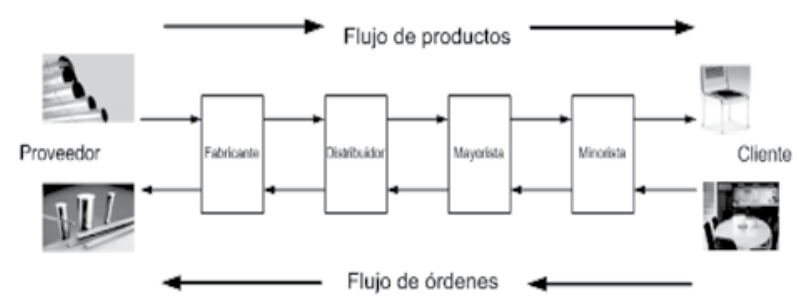

Fuente: elaboración propia

\subsubsection{DESCRIPCIÓN DE LA CONSTRUCCIÓN DEL MODELO}

El desarrollo experimental se realizó implementando una aplicación desarrollada con el software Visual Studio 2003, aplicación que permite tener un mejor control del modelo. Las primeras aproxima- ciones del experimento se realizaron en forma manual, usando una variación del tablero tradicional del juego de la cerveza.

El modelo se construyó teniendo como plataforma una red de computadoras bajo el sistema operativo Microsoft Windows XP y una arquitectura de comunicación soportada bajo TCP/IP, teniendo en cuenta los siguientes programas:

- Herramienta de desarrollo: Visual Studio .NET 2003.

- Lenguaje de programación: Visual Basic .NET.

- Base de Datos: Microsoft Access 2003.

- Net Framework 1.1.

\subsubsection{INFORMACIÓN GENERAL DEL MODELO}

Esta solución cuenta con dos aplicativos (servidor y cliente), divididos en tres capas básicas:

1. Capa de usuario: formularios en los cuales el participante ingresa y visualiza los datos.

2. Capa de negocio: rutinas con la funcionalidad lógica del aplicativo.

3. Capa de datos: base de datos en Microsoft Access 2003.

Para la comunicación entre la aplicación cliente con el servidor se ha utilizado .NET Remoting como tecnología de acceso a objetos remotos.

Esta aplicación puede se extendida para su uso vía Web, teniendo como medio de comunicación el Internet, para se necesitó:

- Un dominio Web.

- Un hosting para su publicación en la Web.

\subsubsection{APLICACIÓN A NIVEL USUARIO}

Constituida por los formularios y controles de interface del usuario y permite interactuar al usuario con el aplicativo.

Figura 5. Diagrama de componentes

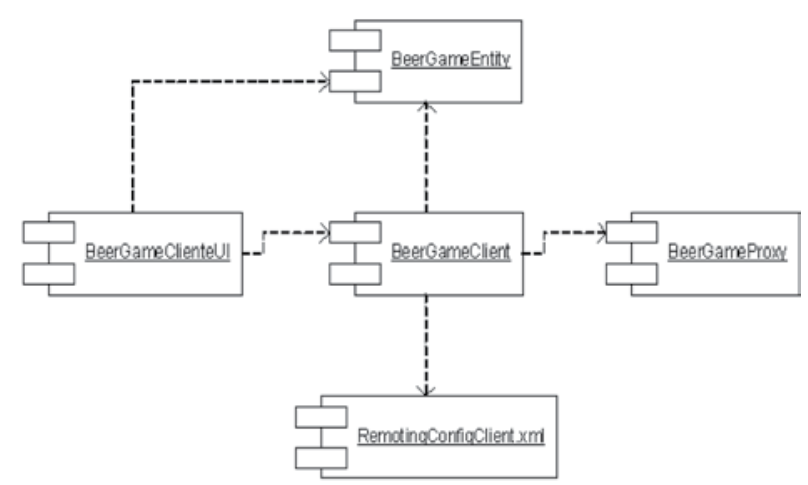

Fuente: elaboración propia 
La aplicación a nivel de usuario tiene los siguientes puntos principales:

1. Pantalla principal

2. Registro de las órdenes

En el cual se representan los siguientes escenarios:

- Escenario 1: Participantes independientes.

- Escenario 2: El participante visualiza la demanda del cliente.

- Escenario 3: Visibilidad de la información.

- Escenario 4: Coordinación.

Figura 6. Pantalla de aplicación usuario

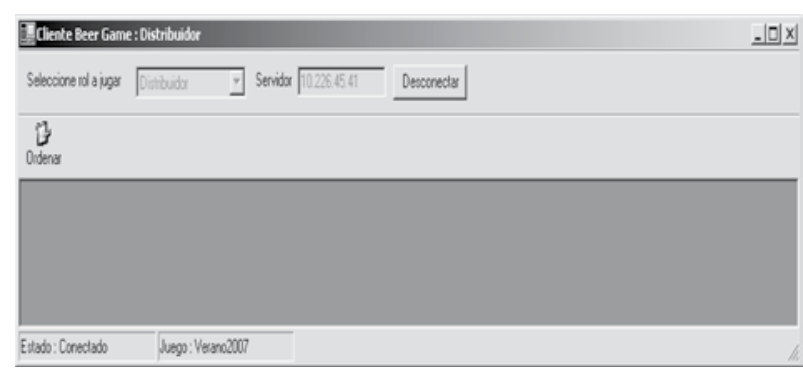

Fuente: elaboración propia.

\section{PROYECTOS}

BeerGameUl.vbproj: Contiene los formularios, módulos, controles y librerías necesarios para la generación del archivo ejecutable (exe)

Figura 7- Módulo de BeerGameClienteUI

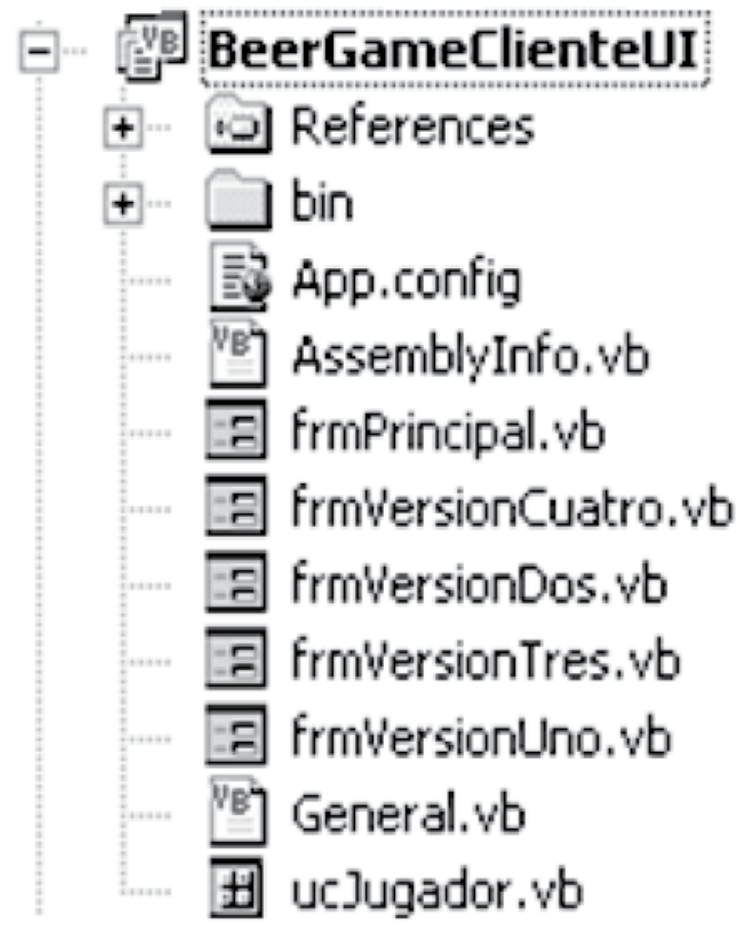

Fuente: elaboración propia
BeerGameClienteProxy.vbproj: Conjunto de archivos que contienen llamadas a los componentes remotos de negocio.

Figura 8. Módulo de BeerGameClienteProxy

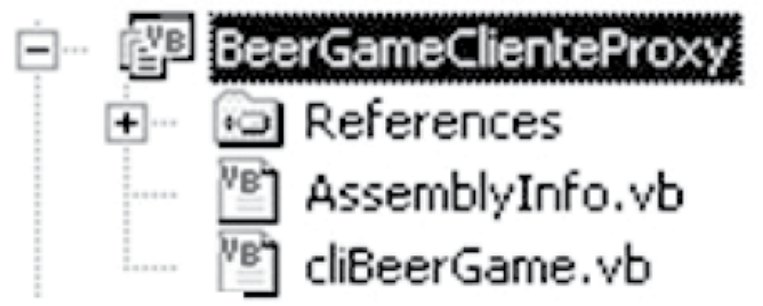

Fuente: elaboración propia

BeerGameClienteUl.exe: Archivo ejecutable, el cual es instalado en el cliente.

\subsubsection{APLICACIÓN A NIVEL SERVIDOR:}

Implementada con una lógica de negocio que recibe los las peticiones enviadas de la aplicación de usuario, generando los flujos de información y registrándolos en la base de datos; también cuenta con un conjunto de formularios utilizado para la creación del juego, ingreso de demanda y monitoreo del juego.

Figura 9. Diagrama de componentes

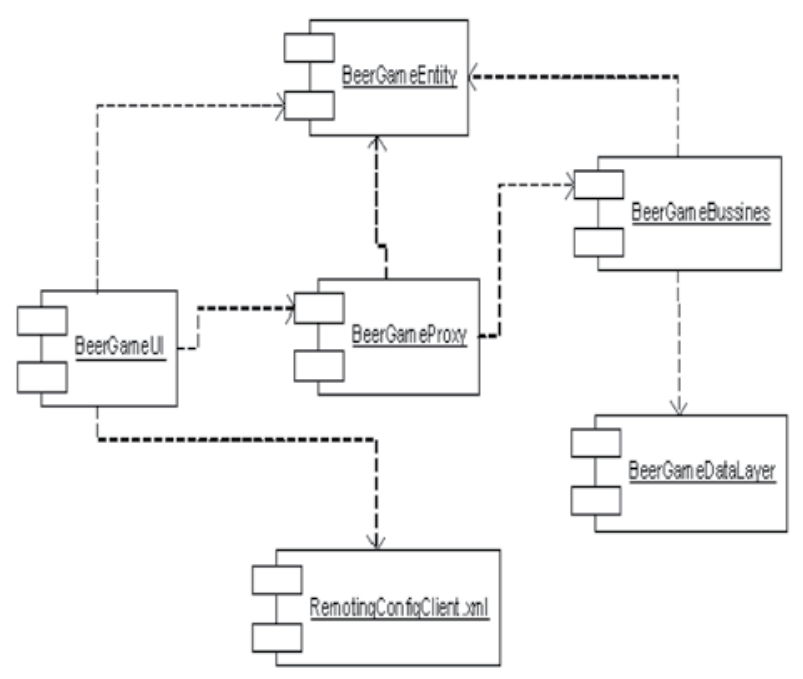

Fuente: elaboración propia

La aplicación a nivel del servidor tiene las siguientes opciones:

- Administración.

- Creación y configuración

- Generación de la demanda.

- Inicialización del servidor.

- Opción monitorear. 
Figura 10. Estado del distribuidor

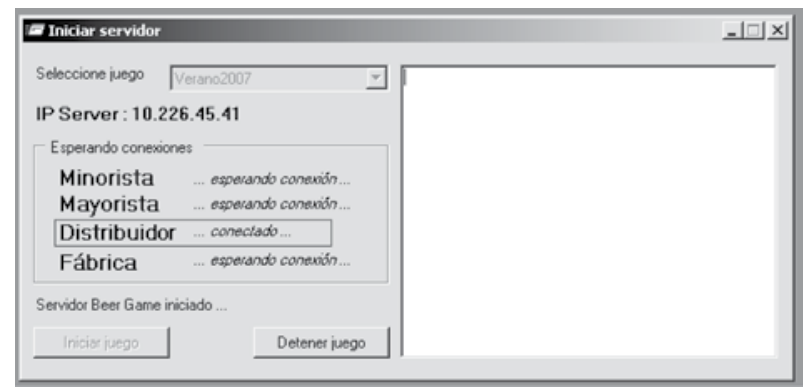

Fuente: elaboración propia

\section{PROYECTOS:}

BeerGameProxy.vbproj: Contiene la definición de las interfaces, clases y métodos que serán accesibles remotamente.

Figura 11. Gráfica de BeerGameProxy

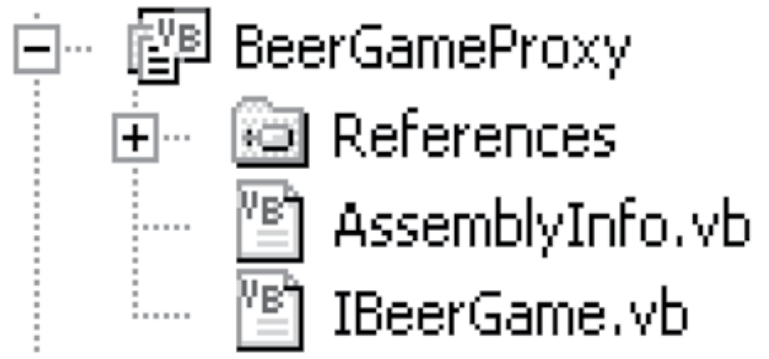

Fuente: elaboración propia

BeerGameBussines.vbproj: Contiene la definición de la lógica del juego.

Figura 12. Gráfica de BeerGameBussines

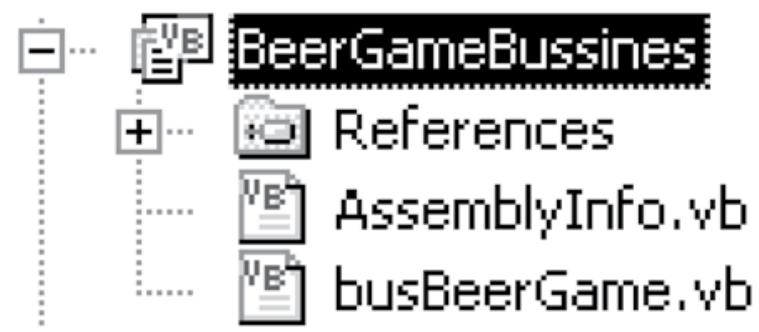

Fuente: elaboración propia

BeerGameDataLayer.vbproj: Contiene las llamadas a la base de datos.

Figura 13. Gráfica de BeerGameDataLayer

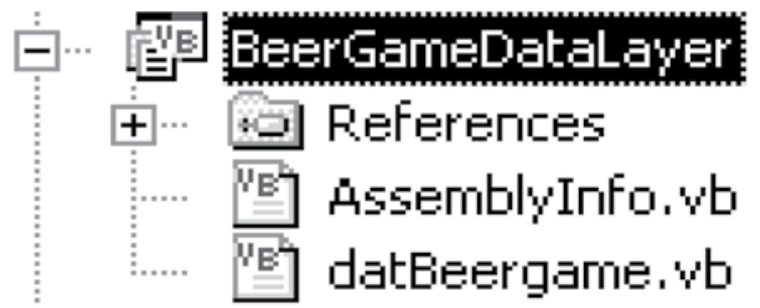

Fuente: Elaboración propia
BeerGameEntity.vbproj: Contiene las estructuras básicas de objetos. Esta estructura es referenciada por todas las aplicaciones (negocio, datos, usuario).

Figura 14. Gráfica de BeerGameEntity

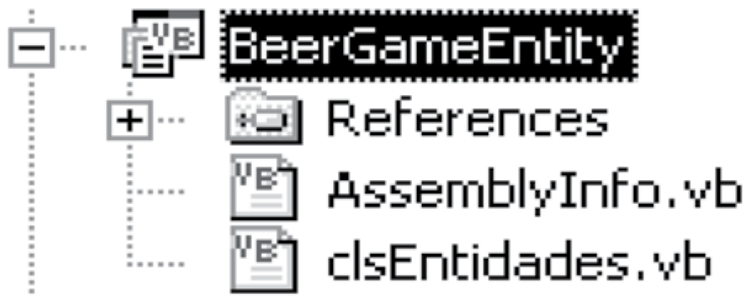

Fuente: elaboración propia

BeerGameUl.vbproj: Contienen los formularios, módulos, controles y librerías necesarios para la generación del archivo ejecutable.

Figura 15. Gráfica de BeerGameUI

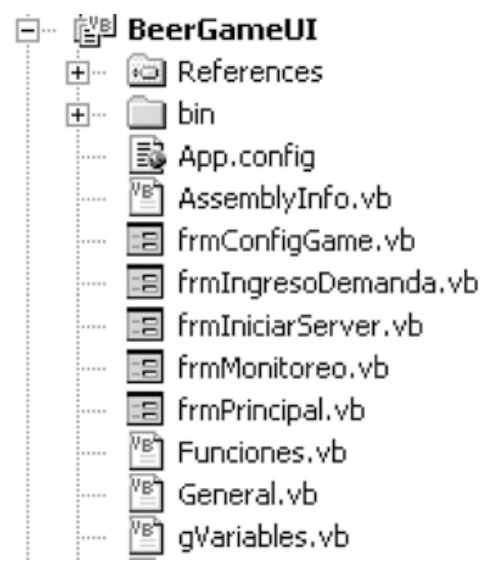

Fuente: elaboración propia

BeerGameUl.exe: Archivo ejecutable instalado en el servidor.

\subsubsection{BASE DE DATOS}

Son un conjunto de tablas relacionales que almacenan los datos estructurados, permitiendo su fácil consulta, registro y modificación. Para esta aplicación se ha utilizado MS Access 2003.

Figura 16. Gráfica de base de datos

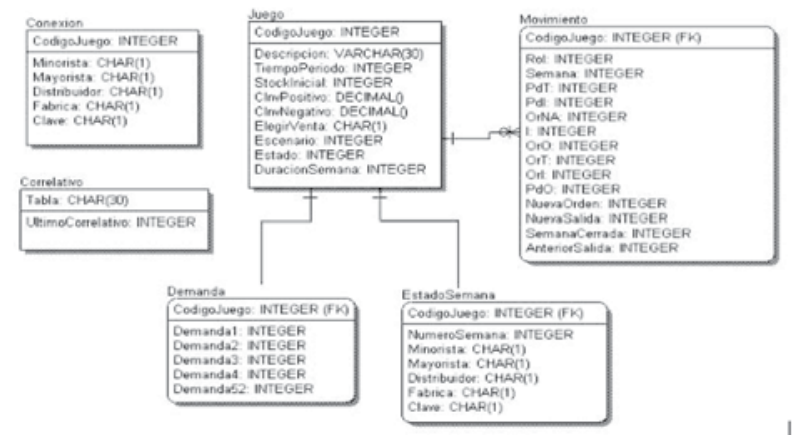

Fuente: elaboración propia. 


\subsection{RESULTADOS EXPERIMENTALES}

\subsubsection{ESCENARIO 1: PARTICIPANTES INDEPENDIENTES}

Los resultados muestran un manejo adecuado de las órdenes y el inventario del minorista en las primeras semanas, pero debido a una falta de productos en su inventario genera un aumento de las órdenes a partir de la décima semana.

Figura 17. Orden con participantes independientes

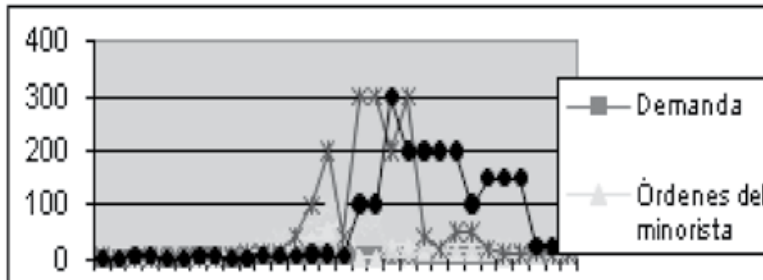

1357911131517192123252729

Fuente: elaboración propia

Figura 18. Gráfica de valor individual

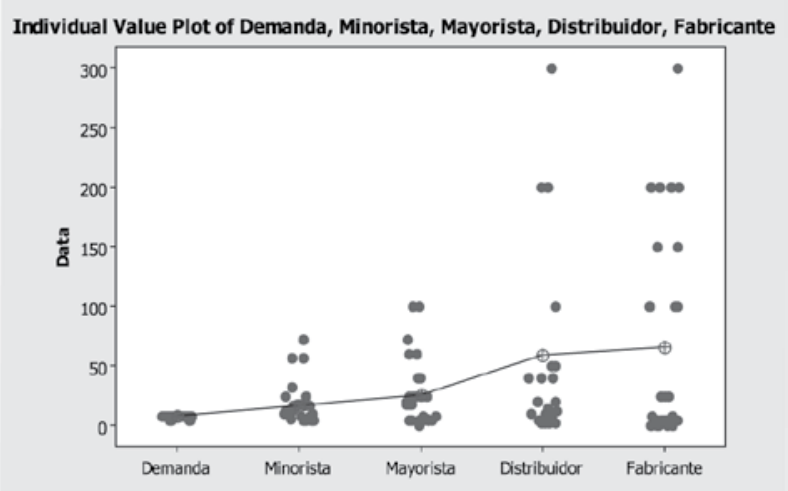

Fuente: elaboración propia

Figura 19. Gráfica de cajas

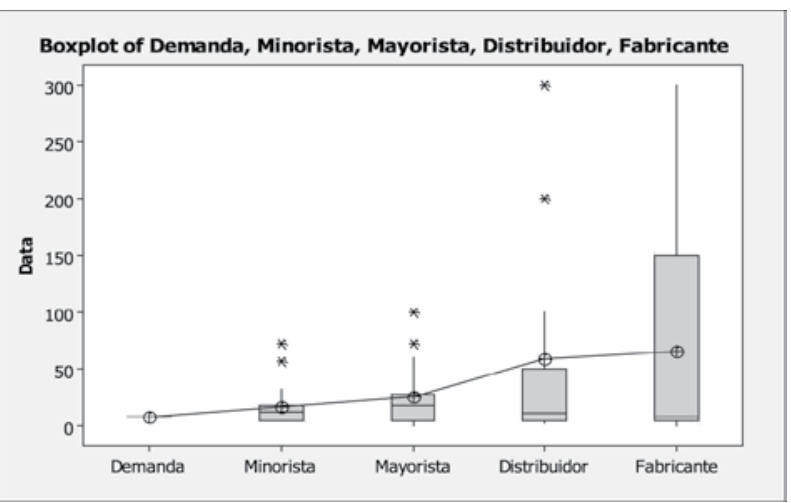

Fuente: elaboración propia

\subsubsection{ESCENARIO 2: DEMANDA DEL CLIENTE FINAL CO- NOCIDA}

Los resultados muestran que el sistema inicialmente se mantiene en equilibrio pero se genera una oscilación y la amplificación de las órdenes desde la octava semana, a pesar del conocimiento de la demanda.

Figura 20. Orden con la demanda final conocida.

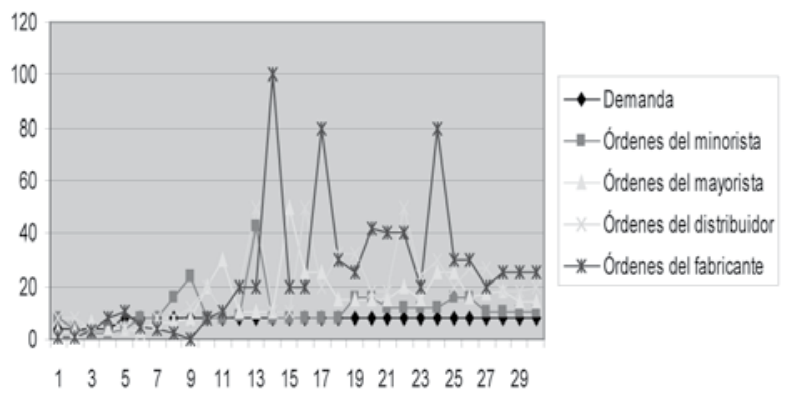

Fuente: elaboración propia

Figura 21. Gráfica de valor individual

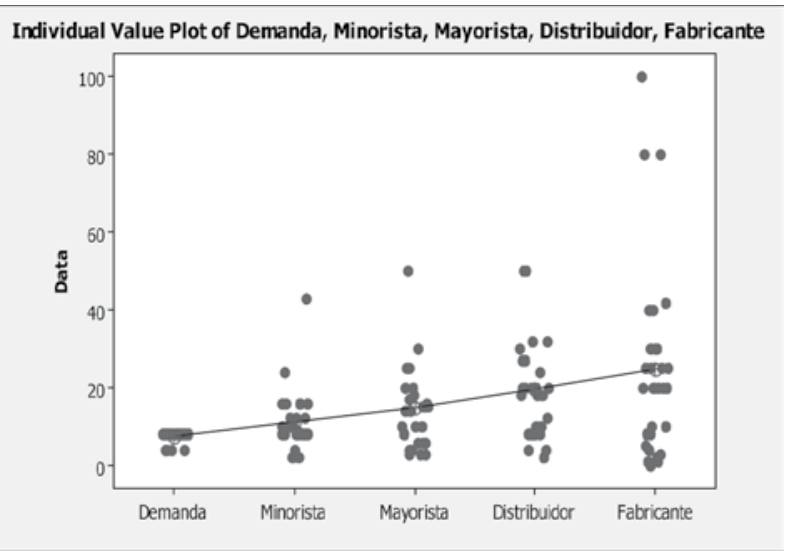

Fuente: elaboración propia

Figura 22. Gráfica de cajas

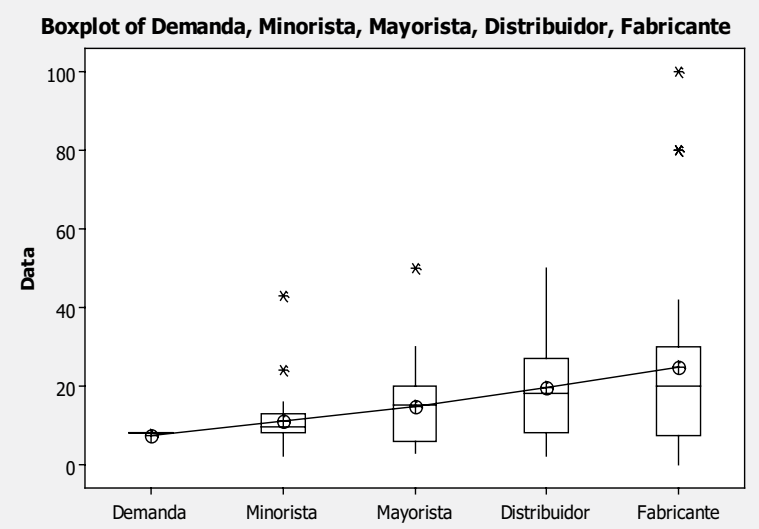

Fuente: elaboración propia

\subsubsection{ESCENARIO 3: VISIBILIDAD DE LA INFORMACIÓN}

Los resultados muestran que con la visualización del flujo de las órdenes y el flujo de los productos se reduce la variabilidad de las órdenes y los cambios bruscos, generando una estabilidad en todo el sistema. 
Figura 23. Orden con visibilidad de información

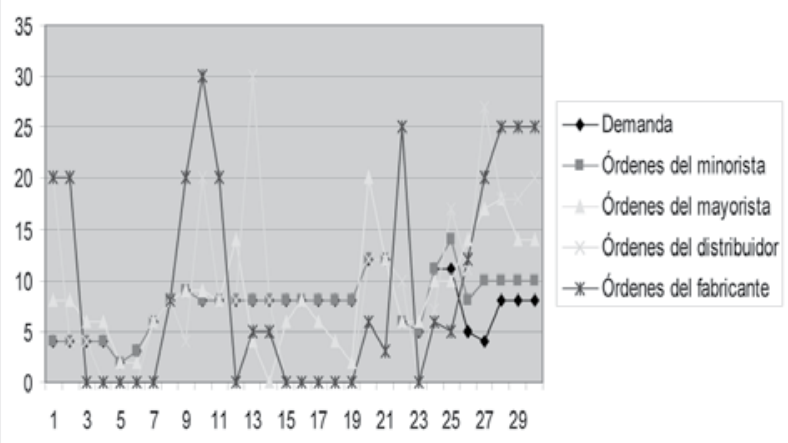

Fuente: elaboración propia

Figura 24. Gráfica de valor individual

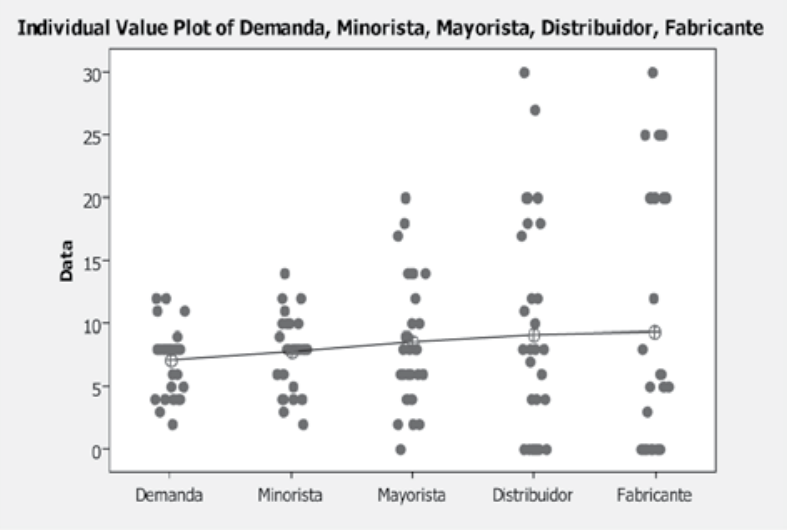

Fuente: elaboración propia

Figura 25. Gráfica de cajas

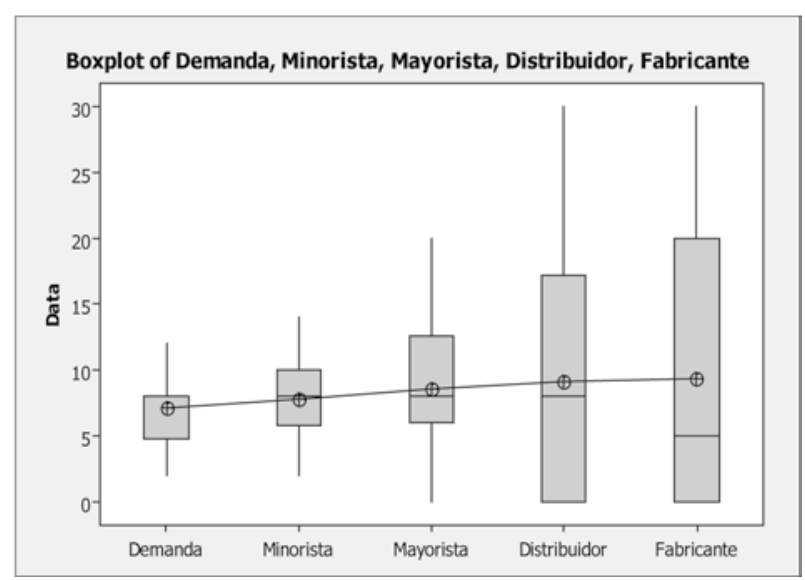

Fuente: elaboración propia.

\subsubsection{ESCENARIO 4: COORDINACIÓN}

El escenario busca eliminar la variabilidad y amplificación de las órdenes con la coordinación. La demanda es similar al escenario anterior con valores desde 2 a 12 .

El escenario muestra un ambiente estable desde las primeras semanas con una reducida amplificación y variabilidad de las órdenes.
Figura 27. Órdenes con coordinación

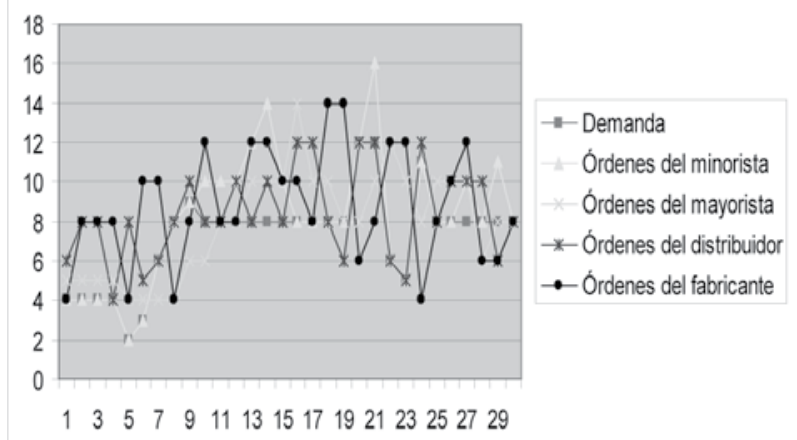

Fuente: elaboración propia

Figura 28. Gráfica de valor individual

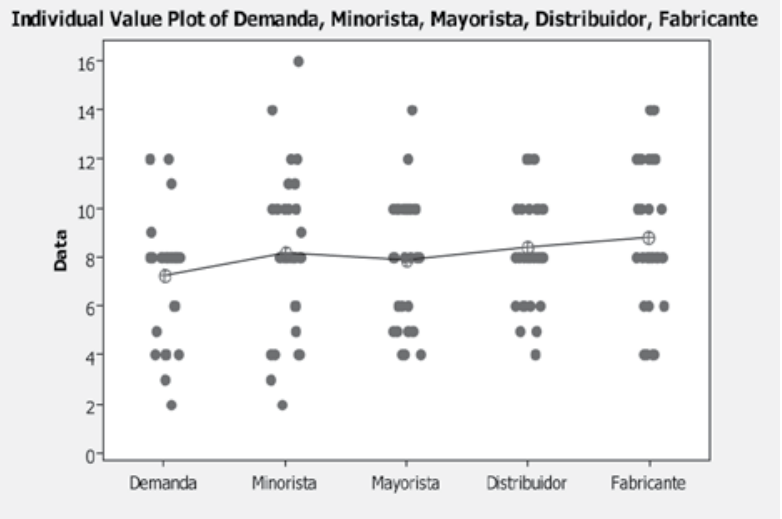

Fuente: elaboración propia

Figura 29. Gráfica de cajas

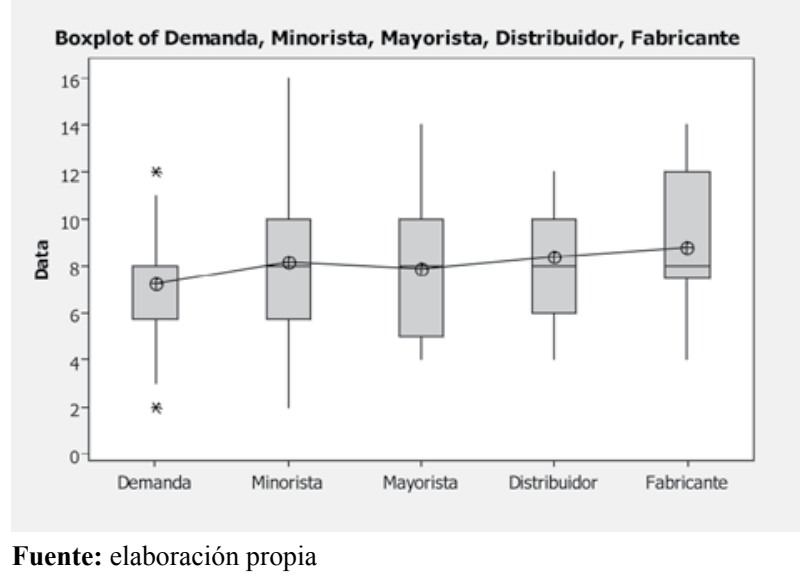

\subsection{ANÁLISIS DE LOS RESULTADOS}

Se muestra una tendencia a reducir los costos totales conforme se aumenta el nivel de información en cada participante. Los escenarios 3 y 4 , visibilidad y coordinación, muestran una reducción de los costos de manera considerable.

Se muestra también que la coordinación puede ser una fuente de incertidumbre si no hay confianza entre los participantes. 
Los escenarios de visibilidad y coordinación reducen en forma significativa la variabilidad de las órdenes. La coordinación genera una estabilidad en las órdenes generadas.

El experimento demuestra que conforme se aumenta el nivel de información entre los participantes, se reduce la amplitud y la variabilidad de las órdenes, teniendo un efecto directo en el costo del total que al final incide en el precio que tiene que pagar el cliente por el producto.

\section{EVALUACIÓN DE UNA EMPRESA METAL ME- CANICA}

La investigación práctica se aplicará en una empresa típica del sector metal mecánico. La clasificación CIIU Rev. $3.1^{4}$, industria de fabricación de muebles metálicos.

\subsection{CONDICIONES DE LA EMPRESA}

- La empresa trata de mantener los costos de producción competitivos debido a que el mercado es muy sensible al precio.

- La selección de la materia prima y el proceso productivo se realiza de acuerdo con los requerimientos de los clientes. Un punto importante es la percepción de los productos como de calidad y durable.

- La entrega de los productos se realiza por una red de distribución propia. En momentos de alta demanda se contrata el servicio de entrega.

- El departamento de producción tiene como prioridad la entrega de los productos en el plazo indicado.

- La planta de producción es flexible a los cambios de la demanda. La planta de producción puede afrontar cambios por el aumento o disminución de la demanda.

- La estructura de producción es flexible para ofrecer una gran variedad de productos personalizados a sus clientes.

- La introducción de nuevos productos se hace con relativa rapidez.

\subsection{DESCRIPCION DEL PROCESO}

Los productos son elaborados según su patrón de flujo, teniendo como procesos principales: corte, lavado, soldado, pintado y ensamble.

\subsection{DESCRIPCIÓN DEL PROBLEMA A ESTUDIAR}

La información proporcionada por la empresa muestra que las solicitudes de productos genera- das por los clientes, en las tiendas minoristas, se cumplen inmediatamente con el inventario disponible. Si no se cuenta con el producto, se genera un flujo de órdenes de producción desde el minorista, al centro de distribución y finaliza en el fabricante.

Figura 30. Diagrama de bloques del proceso.

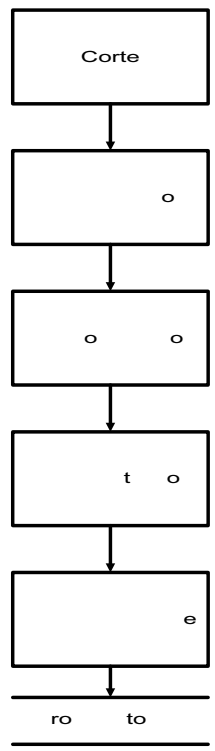

Fuente: elaboración propia

La demanda de los productos no se puede establecer exactamente por las diversas configuraciones proporcionadas y la única forma de conocer el inventario en cada uno de los participantes es por medio de llamadas telefónicas que generan cierto grado de incertidumbre.

Figura 31. Flujo de los participantes
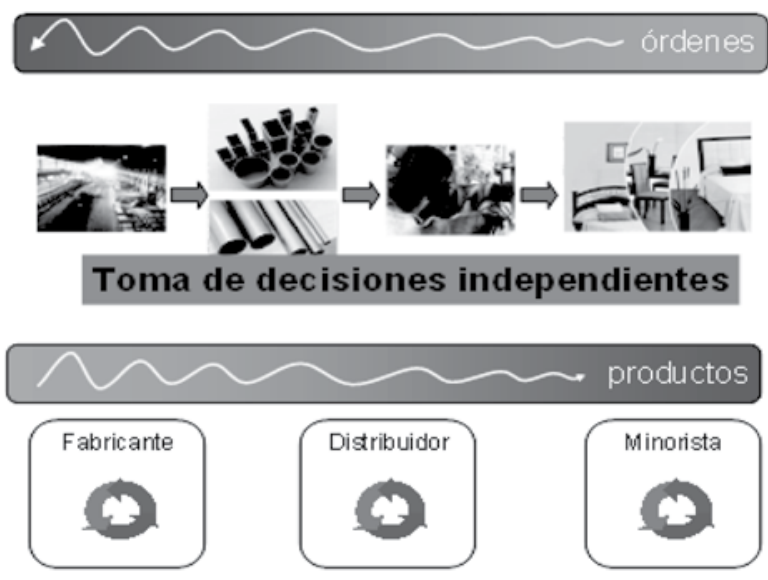

Fuente: elaboración propia

\subsection{DATOS DE LA EMPRESA}

Los datos obtenidos en la empresa se han obtenido teniendo diferentes fuentes de información en cada uno de los participantes:

\footnotetext{
4 Clasificación Industrial Internacional Uniforme
} 
- Minorista: ventas y órdenes de las tiendas.

- Distribuidor: órdenes generadas por el centro de distribución al departamento de producción.

- Fabricante: órdenes de los accesorios para la producción de somier. Basados en el número de accesorios, se obtuvo las órdenes generadas a los proveedores de materia prima.

Los datos de la empresa se obtuvieron de diversas aplicaciones desarrolladas por empresa locales, tenido como base: Microsoft Fox Pro, Microsoft Access y Visual Basic. Además de hojas de cálculo elaboradas por los usuarios de manera individual en: Microsoft Excel y Lotus.

Figura 32. Demanda y órdenes generadas por los participantes en la empresa en estudio - Año 2007:

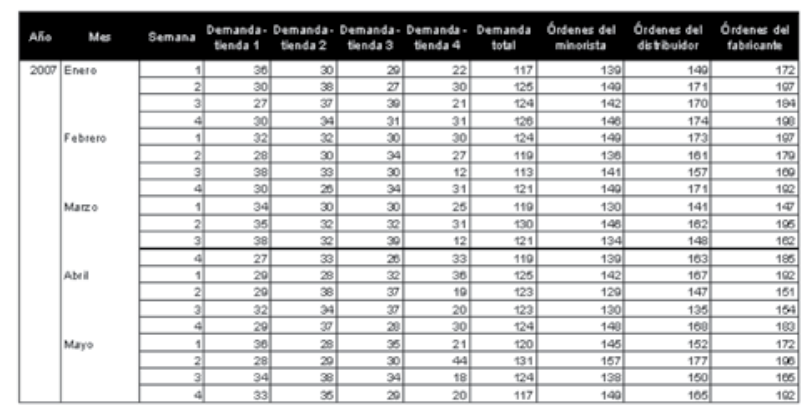

Fuente: elaboración propia, con datos de la empresa

Figura 33. Gráfica de valor individual. Años 2002-2007

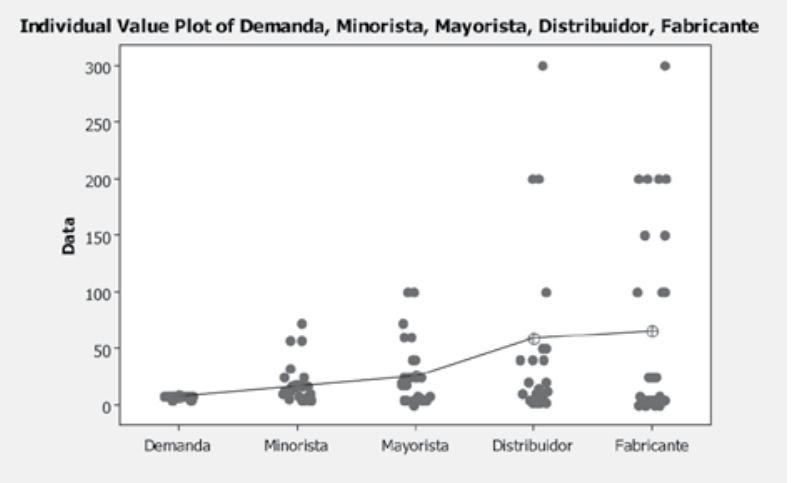

Fuente: elaboración propia

Figura 34. Grafica de cajas. Años 2002-2007

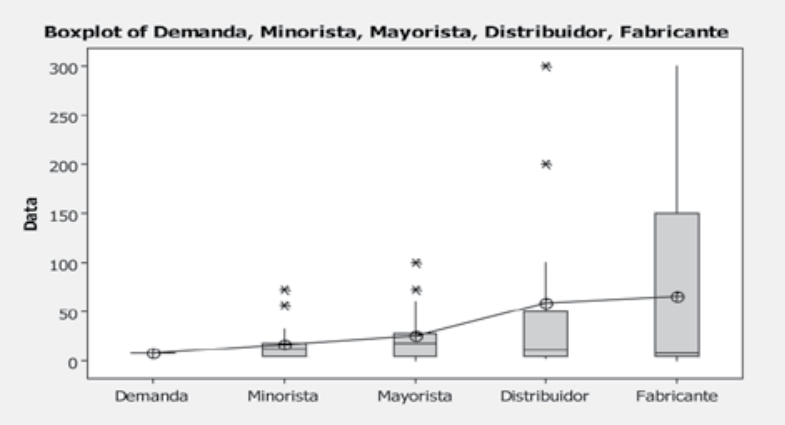

Fuente: elaboración propia
Figura 35. Ordenes semanales de somier - Año 2007

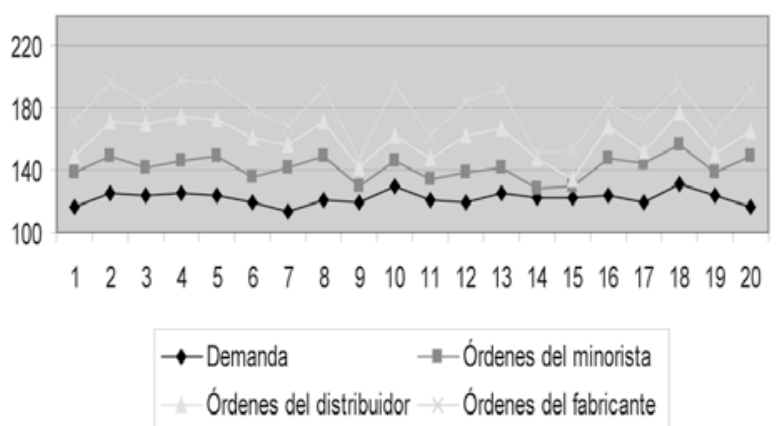

Fuente: elaboración propia, con datos de la empresa

\subsection{ANÁLISIS DE LOS RESULTADOS}

El desarrollo experimental y los datos de la empresa demuestran que el planteamiento teórico y los resultados en diversos ambientes de trabajo son similares para una línea de producción de somier.

La propuesta para este caso es el desarrollo de un proceso de planeamiento colaborativo y un sistema que soporte las operaciones de la empresa como herramienta para:

- Toma de decisiones de manera adecuada.

- Integrar la cadena de suministro, con un adecuado flujo de productos y flujo de información.

- Reducir el nivel de inventario.

- Lograr una estructura empresarial flexible a los cambios.

- Incrementar el nivel de servicio al cliente.

\section{ALTERNATIVAS DE SOLUCIÓN}

La solución se enfoca en la implementación de un sistema que permita reducir la amplificación y la variabilidad de las órdenes con una política de integración de la información y colaboración en los participantes, evitando la distorsión y retraso de la información, incremento de los costos y pérdida de clientes.

Los puntos significativos son:

- Colaboración intensiva de los participantes.

- Compartir la información clave.

- Sincronizar las toma de decisiones sobre capacidades y flujos de materiales, en un ambiente de alta incertidumbre.

- Mejorar la competitividad con el servicio al cliente, incrementando las ventas y reduciendo la cantidad de productos obsoletos e inventarios. 
De acuerdo al diagnóstico inicial de la empresa se plantea dos opciones:

1. Desarrollar en la empresa un sistema individual que integre todas las áreas de la empresa y permita compartir la información de todos los participantes.

2. Implementar un sistema comercial personalizado a las operaciones de la empresa.

Las conversaciones con los directivos de la empresa muestran una preferencia por la segunda opción, por los siguientes motivos:

- Posicionamiento en el mercado.

- Respaldo de la empresa proveedora.

- Posibilidad de personalización de las aplicaciones a los procesos de la empresa y de los participantes.

La determinación de la mejor propuesta de solución se realizó empleando el método de ranking de factores con el análisis de las tres soluciones de acuerdo a los siguientes criterios:

- Facilidad de uso.

- Escalabilidad.

- Seguridad.

- Accesibilidad.

- Flexibilidad.

- Colaborativo.

- Factibilidad.

- Inversión.

La aplicación propuesta para la empresa fue un programa de producción local, teniendo en consideración que los factores de mayor importancia son la factibilidad y la inversión, seguidos por la seguridad y facilidad.

\section{CONCLUSIONES Y RECOMENDACIONES}

1. El desarrollo del estudio ha permitido demostrar el efecto de la información en una empresa metal mecánica desde los niveles de participantes independientes hasta la coordinación (de 300 a 14 órdenes como máximo).

2. Los resultados proponen implementar un sistema que permita la transparencia de la información de los participantes para mejorar el manejo de los productos en la cadena de suministro. El efecto se demuestra experimentalmente (escenario 3-Visibilidad de la información) con una reducción de los costos de inventario y los costos de incumplimiento, que al final se transfiere al precio final del producto.
3. La incidencia de la coordinación y el efecto de la información entre los participantes se demuestran con el escenario 3 - Visibilidad de la información y el escenario 4 - Coordinación. La visibilidad de la información tiene mejores resultados en costos, amplificación y variabilidad por las causas conductuales en la toma de decisiones al momento de la coordinación.

4. El efecto de la información se realizó en la línea de producción de la empresa. Es recomendable estudiar el efecto en todas las líneas de la empresa para medir el impacto en toda la organización.

\section{REFERENCIAS}

[1] Ballou, R. (2004). Logística. Administración de la Cadena de Suministro - $5^{\circ}$ edición, Pearson Educación, México D.F., México.

[2] Banks, J. (1996). Discrete Event System Simulation, Prentice Hall, New Jersey, USA.

[3] Chase, R. (2009). Administración de operaciones: producción y cadena de suministros $-12^{\circ}$ edición, McGraw Hill, México D. F., México.

[4] Forrester, J. (1972). Dinámica Industrial, El Ateneo, Buenos Aires, Argentina.

[5] Harrell, C. (1994). Simulation. Made Easy User Guide, Engineering \& Management Press, Georgia, USA.

[6] Kelton, D.(1998). Simulation With Arena, McGraw Hill, Boston, USA.

[7] Kelton, D.(2011). Simio \& simulation: modeling, analysis, applications, McGraw Hill Learning Solutions, New York, USA.

[8] Krajewski, L. (2008). Administración de operaciones: procesos y cadenas de valor $-8^{\circ}$ edición, Pearson Educación, México D.F., México.

[9] Laudon, K. (2008). Sistemas de información gerencial. Administración de la empresa digital - 10 edición, Pearson Educación, México D.F., México.

[10] Morecroft, J. (2000). Modeling for learning organizations, Productivity Press, New York, USA.

[11] Pritsker, A. (1998). Simulation With Visual Slam and Awe Sim, John Wisley\& Son, New York, USA.

[12] Stallings, W. (2004). Computer networking with Internet protocols and technology, Pearson Education, New Jersey, USA.

[13] Sterman, J. (2000). Business dynamics : systems thinking and modeling for a complex world, Irwin/Mcgraw-Hill, Boston, USA. 